\title{
H3K27M-mutant diffuse midline glioma with extensive intratumoral microthrombi in a young adult with COVID-19-associated coagulopathy
}

\author{
Matthew Pun ${ }^{1,2}$. James Haggerty-Skeans $s^{1,2}$. Drew Pratt ${ }^{2}$ - Yelena Fudym ${ }^{2}$. Wajd N. Al-Holou ${ }^{3}$. \\ Sandra Camelo-Piragua ${ }^{2} \cdot$ Sriram Venneti $^{2,4}$ (i)
}

Received: 7 June 2020 / Revised: 21 June 2020 / Accepted: 21 June 2020 / Published online: 29 June 2020

(c) Springer-Verlag GmbH Germany, part of Springer Nature 2020

The emergence of the novel coronavirus disease 2019 (COVID-19) presents unique challenges for oncology patients. In addition to effects on access to care, initial studies have demonstrated an increased risk of infection and death in cancer patients [5]. COVID-19 has also been linked to hypercoagulability and elevated cerebrovascular accidents rates $[3,6,9]$; however, little is known regarding the impact of COVID-19-associated coagulopathy on tumor biology. Malignancies are well associated with hypercoagulation, varying by age and tumor type. Brain tumors are associated with high risk for thromboembolism in adults but not in children $[1,8]$. Here, we discuss the case of a young adult with a thalamic high-grade glioma, COVID-19-associated

Matthew Pun and James Haggerty-Skeans have equally contributed to this work.

Electronic supplementary material The online version of this article (https://doi.org/10.1007/s00401-020-02184-0) contains supplementary material, which is available to authorized users.

Sandra Camelo-Piragua

sandraca@med.umich.edu

Sriram Venneti

svenneti@med.umich.edu

Matthew Pun

punm@med.umich.edu

James Haggerty-Skeans

jarha@med.umich.edu

1 Medical Scientist Training Program, University of Michigan Medical School, Ann Arbor, MI 48109, USA

2 Department of Pathology, University of Michigan Medical School, Ann Arbor, MI 48109, USA

3 Department of Neurosurgery, University of Michigan, Ann Arbor, MI 48109-5338, USA

4 University of Michigan, 3520E MSRB 1, 1150 West Medical Center Drive, Ann Arbor, MI 41804, USA coagulopathy, and histopathologic evidence of extensive vascular thrombosis within the tumor microenvironment.

A 21-year-old woman presented with headaches, blurry vision, and vomiting. Her neurologic examination showed no abnormalities, and the patient was diagnosed with tension-type headache. Two months later, she experienced a syncopal episode. Head CT demonstrated a large $(4.3 \mathrm{~cm})$ supratentorial thalamic mass causing obstructive hydrocephalus. She subsequently decompensated from herniation and underwent emergent ventricular drain placement. Post-operative head CT and MRI demonstrated successful drain placement, but exhibited marked hemorrhage in the tumor and ventricles, with displacement of the septum pellucidum (Figs. 1a, b and Supplementary Figure. 1a, online resource). The patient tested positive for SARS-CoV-2 by nasopharyngeal swab and exhibited classic findings of COVID-19-associated coagulopathy including elevated D-dimer levels (Fig. 1c), without changes in prothrombin or activated-partial-thromboplastin time (Supplementary Table 1, online resource) [3]. She exhibited no respiratory symptoms, and a chest radiograph demonstrated no evidence of lung disease.

Biopsy demonstrated a high-grade glial tumor (Fig. 1d) expressing GFAP (Fig. 1e). Tumor cells showed H3K27M staining (Fig. 1f), reduced H3K27me3, and high Ki-67 (Supplementary Figure.1b-c, online resource). Notably, the tumor showed multiple areas of hemorrhage, thrombosed blood vessels, and microthrombi with fibrin deposition, confirmed by a PTAH stain (Figs. 1g-1 and S1d-1, online resource). The tumor exhibited parenchymal and perivascular macrophage infiltration (Figs. 1m-n) and rare CD4-positive T cells (Fig. 1o). SARS-CoV-2 in situ hybridization was negative (Supplementary Figures. 1m-n, online resource).

Microthrombi in IDH-WT glioblastomas are associated with necrosis, hypoxic niches, and development of treatment resistance. Intriguingly, IDH-mutant gliomas show lower 

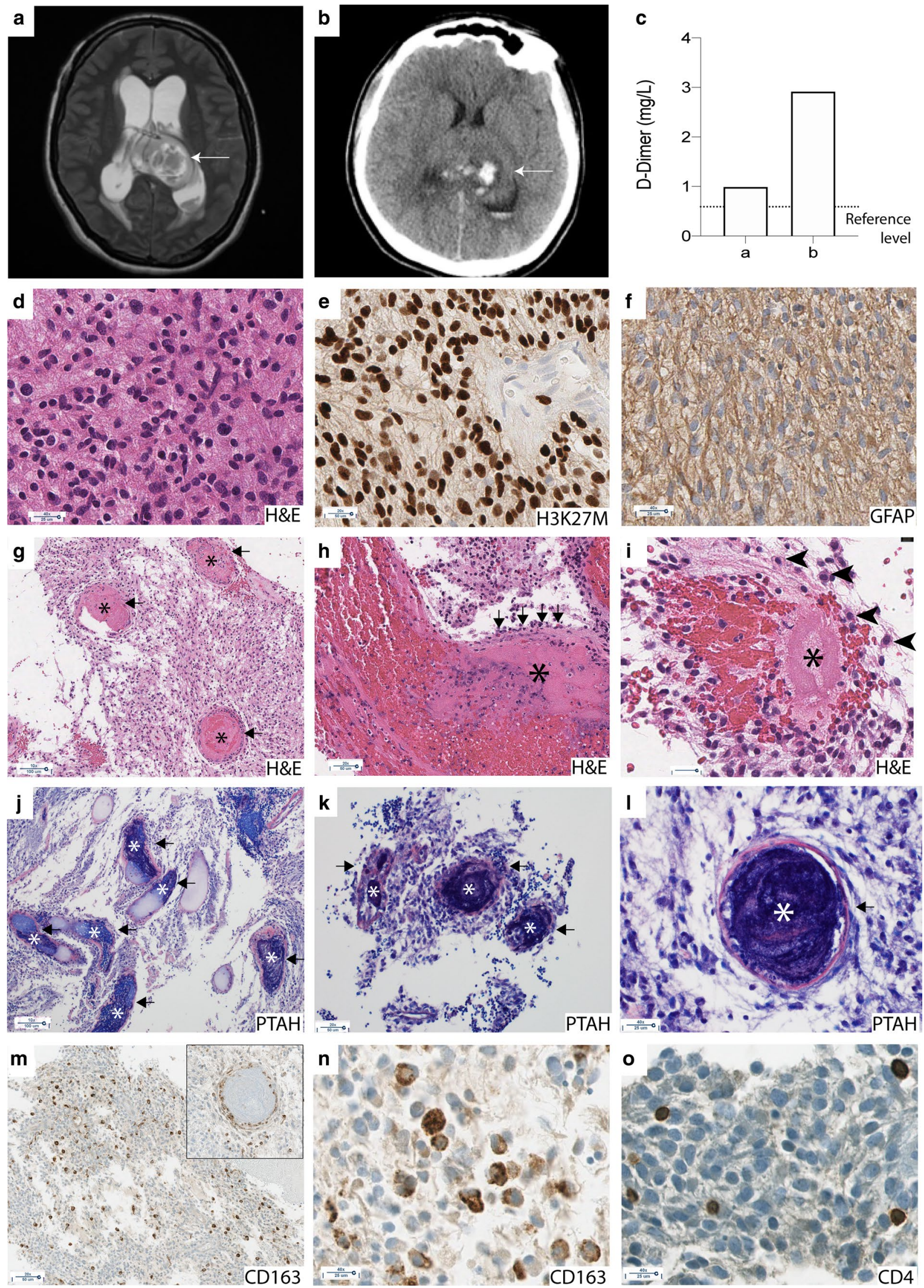
४Fig. 1 Radiologic images, D-dimer levels, and neuropathologic features. a, b Horizontal MRI (a) and CT (b) images showing a thalamic mass with midline shift. Arrows indicate hemorrhage. $\mathbf{c}$ D-dimer levels measured on 2 consecutive days ( $a$ and $b$; dotted line represents upper limit of reference value). d Representative H\&E image demonstrating pleomorphic tumor cells. e, f Immunohistochemistry demonstrating GFAP (e) and H3K27M using a mutant-specific antibody (f) in tumor cells. $\mathbf{g}-\mathbf{l}$ H\&E images indicating multiple thrombosed blood vessels with hemorrhage (arrows). Asterisk shows eosinophilic amorphous fibrin deposits. Arrowheads indicate macrophages $(\mathbf{g}-\mathbf{i})$. Intravascular fibrin deposits were confirmed by PTAH staining $(\mathbf{j}-\mathbf{l})$. m-o Immunohistochemical stains for CD163 highlighting parenchymal $(\mathbf{j}-\mathbf{k})$ and perivascular macrophages (j, inset) and CD4-positive lymphocytes (o)

frequencies of microthrombi [7]. Because the frequency of thrombosis in H3K27M gliomas is unknown, we evaluated ten cases, finding far fewer microthrombi (mean $=2.9$, median $=0.5$ ) than the current case $(>40$, Supplementary Figure. 1o, online resource). Moreover, brainstem gliomas in children and young adults have low frequencies of intratumoral hemorrhage [2].

Recent reports suggest a unique predisposition of the brain to thrombosis in COVID-19 infections attributed to a cytokine storm syndrome $[4,6]$. Given the poor association between intratumoral microthrombi and overall prognosis in gliomas, our case raises a critical question concerning the impact of COVID-19-associated coagulopathy on tumor biology, the immune microenvironment, and, in this case, the patient's clinical course. Our case poses the hypothesis that COVID-19-associated coagulopathy could affect longterm tumor behavior and disease progression in cancer in a manner yet unrecognized.

\section{References}

1. Athale U, Siciliano S, Thabane L, Pai N, Cox S, Lathia A et al (2008) Epidemiology and clinical risk factors predisposing to thromboembolism in children with cancer. Pediatr Blood Cancer 51:792-797. https://doi.org/10.1002/pbc.21734

2. Broniscer A, Laningham FH, Kocak M, Krasin MJ, Fouladi M, Merchant TE et al (2006) Intratumoral hemorrhage among children with newly diagnosed, diffuse brainstem glioma. Cancer 106:1364-1371. https://doi.org/10.1002/cncr.21749

3. Colling ME, Kanthi Y (2020) COVID-19-associated coagulopathy: an exploration of mechanisms. Vasc Med. https://doi. org/10.1177/1358863X20932640

4. Coolen T, Lolli V, Sadeghi N, Rovaï A, Trotta N, Taccone FS et al (2020) Early postmortem brain MRI findings in COVID-19 nonsurvivors. Neurology. https://doi.org/10.1212/WNL.0000000000 010116

5. Mehta V, Goel S, Kabarriti R, Cole D, Goldfinger M, AcunaVillaorduna A et al (2020) Case fatality rate of cancer patients with covid-19 in a New York hospital system. Cancer Discov. https://doi.org/10.1158/2159-8290.cd-20-0516

6. Reichard RR, Kashani KB, Boire NA, Constantopoulos E, Guo Y, Lucchinetti CF (2020) Neuropathology of COVID-19: a spectrum of vascular and acute disseminated encephalomyelitis (ADEM)-like pathology. Acta Neuropathol 140:1-6. https://doi. org/10.1007/s00401-020-02166-2

7. Unruh D, Schwarze SR, Khoury L, Thomas C, Wu M, Chen L et al (2016) Mutant IDH1 and thrombosis in gliomas. Acta Neuropathol 132:917-930. https://doi.org/10.1007/s00401-016-1620-7

8. Walker AJ, West J, Card TR, Crooks C, Grainge MJ (2012) Rate of venous thromboembolism by cancer type compared to the general population using multiple linked databases. Thromb Res 129:S155-S156. https://doi.org/10.1016/S0049-3848(12)70036-1

9. Zhou F, Yu T, Du R, Fan G, Liu Y, Liu Z et al (2020) Clinical course and risk factors for mortality of adult inpatients with COVID-19 in Wuhan, China: a retrospective cohort study. Lancet 395:1054-1062. https://doi.org/10.1016/s0140-6736(20)30566-3

Publisher's Note Springer Nature remains neutral with regard to jurisdictional claims in published maps and institutional affiliations. 